\title{
Modeling of an On-Chip Power/Ground Meshed Plane Using Frequency Dependent Parameters
}

\author{
Chulsoon Hwang $\cdot$ Kiyeong Kim $\cdot$ Jun So Pak $\cdot$ Joungho Kim
}

\begin{abstract}
This paper proposes a new modeling method for estimating the impedance of an on-chip power/ground meshed plane. Frequency dependent $R, L$, and $C$ parameters are extracted based on the proposed method so that the model can be applied from DC to high frequencies. The meshed plane model is composed of two parts: coplanar multi strip (CMS) and conductor-backed CMS. The conformal mapping technique and the scaled conductivity concept are used for accurate modeling of the CMS. The developed microstrip approach is applied to model the conductor-backed CMS. The proposed modeling method has been successfully verified by comparing the impedance of RLC circuit based on extracted parameters and the simulated impedance using a 3D-field solver.
\end{abstract}

Key words: Conformal Mapping, On-Chip Meshed Plane, Power Distribution Network, Simultaneous Switching Noise.

\section{Introduction}

Recently, communication, computing, and sensing systems have incorporated noise-sensitive analog RF circuits, and noisy digital circuits into a single chip/package for small form factors and high performance. In this type of mixed-mode system, however, the simultaneous switching noise (SSN) caused by high-speed digital circuits not only affects digital functionality but also degrades the receiver sensitivity and reliability of neighboring analog and RF circuits [1], [2].

The amount of SSN is determined by the amount of current consumption of the circuits and the impedance profile of the power distribution network (PDN) seen from the circuits. An efficient way to reduce the amount of SSN is to design a PDN that has low impedance at the switching frequency and its harmonics. Therefore, estimation of the impedance properties of the PDN is important for the reliable operation of the system. As the operating frequency exceeds $\mathrm{GHz}$ levels, the highfrequency performance of the PDN should also be taken into consideration.

For on-chip PDNs in high-speed systems, a meshed plane, as shown in Fig. 1, has been widely adopted because of its low inductance. The on-chip power/ground meshed plane has been extensively analyzed [3] [5]. However, it is difficult to apply previous works to meshed planes that are fabricated with standard CMOS processes because they are not compatible with the SPICE

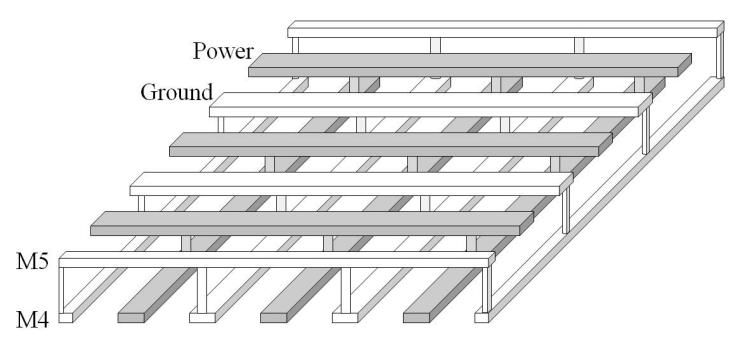

Fig. 1. Conventional on-chip power/ground meshed plane composed of two metal layers.

simulator [3]. They are also not suitable for a standard CMOS process because of the vertical configurations; the metal thickness and the dielectric layer stack-up are different. Consequently, the applicable frequency range of the works is limited to a very narrow frequency range in a standard CMOS process [4], [5].

This paper provides details about the modeling of an on-chip meshed plane on low-resistivity silicon substrates. Quasi-static analysis using conformal mapping and the scaled conductivity concept are applied to calculate the frequency dependent parameters of the coplanar multi strip (CMS) structure. For a conductor-backed CMS, the developed microstrip approaches are simplified to suit the physical dimensions of the targeted structure and are applied to extract parameters. The impedance profiles of the on-chip meshed plane, estimated by a circuit simulation that is composed of extracted $R, L$, and $C$ parameters, were compared with the results of a $3 \mathrm{D}$ -

Manuscript received March 15, 2011 ; revised June 22, 2011. (ID No. 20110315-011J)

Department of Electrical Engineering, KAIST, Dajeon, Korea.

Corresponding Author : Joungho Kim (e-mail : joungho@ee.kaist.ac.kr) 
field solver and showed an excellent correlation over a wide range of frequencies.

\section{Modeling Procedure}

In a conventional on-chip meshed plane, as shown in Fig. 1, the power and ground lines are interdigitated and equipotential lines across different layers that are connected through vias. The meshed structure is periodic, such that a unit cell analysis is applied to model the entire meshed plane. The repeated pattern is defined as a unit cell by placing the side magnetic walls at the edge of the repeated pattern. Magnetic walls may be placed at any site where there is a zero tangential $\mathrm{H}$-field along an entire place. The unit cell is shown in Fig. 2(a) and its equivalent circuit model is shown in Fig. 2(b). The parameters will be evaluated in section III and section IV . Series impedance, i.e., $R$ and $L$, is a frequency dependent parameter and $C$ is a frequency independent parameter. Because a low-loss substrate is assumed, the conductance $(\mathrm{G})$ is negligible in our frequency range of interest.

The orthogonal lines have zero mutual inductance, since there is no magnetic linkage. Therefore, orthogonal lines can be evaluated independently [6]. Mesh lines in one direction only affect the mesh inductance in the same direction. This behavior is analogous to the properties of mesh resistance. Hence, one-dimensional wave

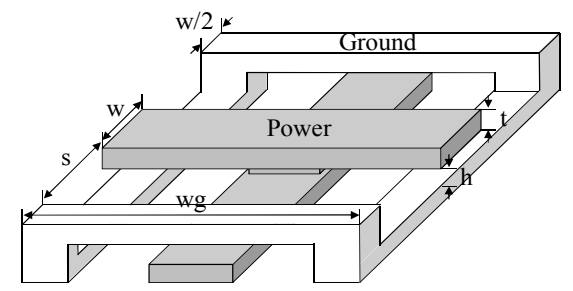

(a)

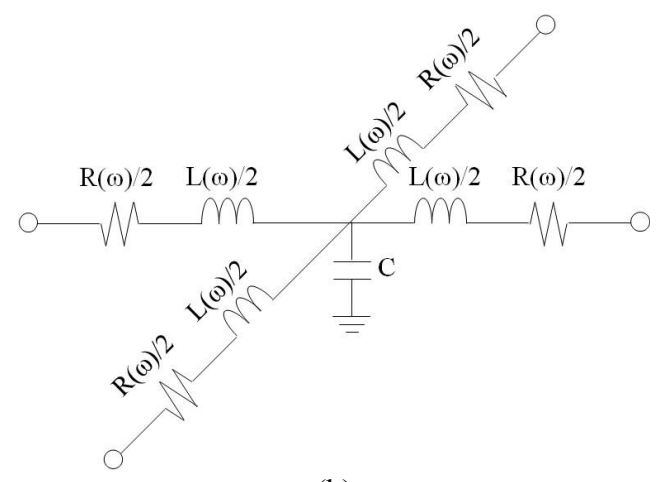

(b)

Fig. 2. (a) Unit cell of the meshed plane for parameter extraction, (b) Equivalent circuit representation of the unit cell.
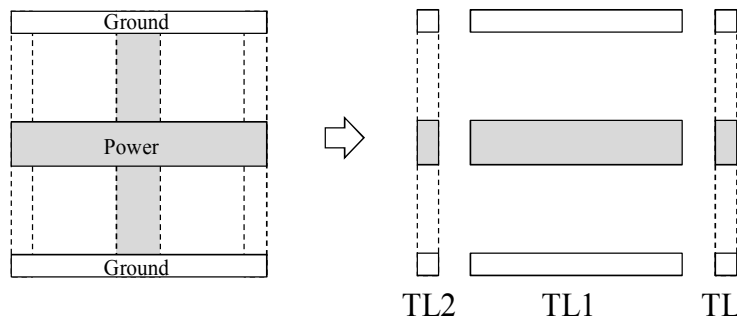

Fig. 3. Unit cell divided into two types of transmission lines in one-dimensional analysis.

propagation is analyzed and then expanded to two-dimensional wave propagation. The one-dimensional unit cell is divided into two cascaded transmissions with a uniform cross section, coplanar multi strip (CMS), and conductor-backed CMS as shown in Fig. 3. Solid lines represent the upper metal layer and dashed lines represent the lower layer.

The cross sections of the two types of transmission line that form the power/ground meshed plane are shown in Fig. 4.

The capacitance of the CMS was evaluated using quasi-static analysis based on conformal mapping. The frequency dependent inductance and resistance were evaluated by applying the scaled conductivity concept to conformal mapping. The conductor-backed CMS was treated as a microstrip line, so the previous equations were applied to evaluate the series impedance and capacitance. The parameters in the microstrip structure were calculated using an empirical equation based on analy-

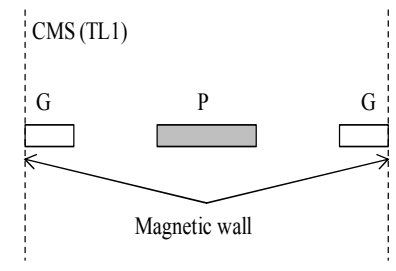

(a) CMS (coplanar multi strip)

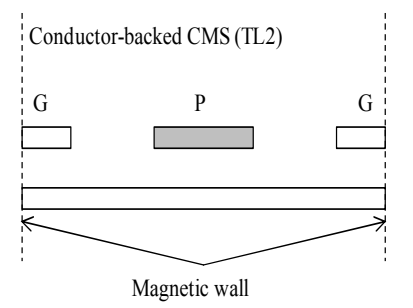

(b) Conductor-backed CMS

Fig. 4. Two types of transmission lines forming the power/ ground meshed plane. 
tical equations. Detailed equations for the CMS are presented in section III and those for the conductor-backed CMS are presented in section VI. The overall $R, L$, and $C$ parameters are given below:

$$
\begin{aligned}
& R(\omega)=(2 s+w) R_{C M S}(\omega)+w R_{\text {Micro }}(\omega) \\
& L(\omega)=(2 s+w) L_{C M S}(\omega)+w L_{\text {Micro }}(\omega) \\
& C=2(2 s+w) C_{C M S}(\omega)+2 w C_{\text {Micro }}
\end{aligned}
$$

where $\mathrm{w}$ is the metal width, and $\mathrm{s}$ is the metal space. $X_{C M S}$ and $X_{\text {Micro }}$ represent parameters per unit length in the CMS-type transmission line and the conductor-backed CMS-type transmission line, respectively.

\section{Coplanar Multi Strip}

Conformal mapping, which has been well established as a useful technique for calculating propagation constants of quasi-TEM transmission lines, was used to evaluate the capacitance. This quasi-TEM analysis is valid until dispersion occurs, which is within our frequency range of interest. When the electric potential on the power strips is $V_{d d}$ and the potential on the ground strips is $0 \mathrm{~V}$, the electric field distribution of CMS would be as shown in Fig. 5, and a magnetic wall can be inserted at the center of the strips because no electric field exists normal to the line connecting the power and ground strips [7]. Because the structure is bilaterally symmetric, a half section is evaluated and then applied to the whole structure.

The boundary condition of CMS is different from a conventional coplanar waveguide (CPW), which was introduced in [8]. Therefore, applying the CPW equations on the CMS may cause errors. For this reason, a dedicated equation for the CMS structure is formulated using the conformal mapping technique.

To apply conformal mapping, the slot between the strips is modeled as a magnetic wall. Thus, the overall capacitance can be evaluated as the sum of the capacitance of the upper and lower half-planes. In Fig. 6, the

\begin{tabular}{|c|c|c|}
\hline$\varepsilon_{\mathrm{I}}=1$ & & Open boundary \\
\hline$\varepsilon_{\mathrm{rl}}$ & $\mathrm{h}_{1}$ & Dielectric \\
\hline$\varepsilon_{12}$ & $\mathrm{~h}_{2}$ & Dielectric \\
\hline$\varepsilon_{\mathrm{T} 3}$ & & Open boundary \\
\hline
\end{tabular}

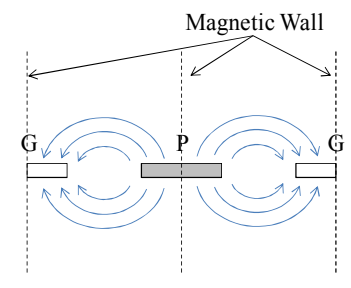

Fig. 5. Electric field of coplanar power/ground strips. (a)

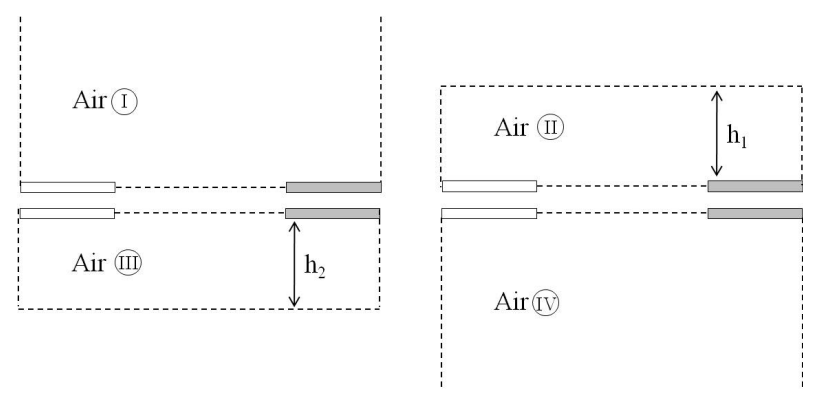

(b)

Fig. 6. (a) A CMS structure with a dielectric-filled layer and (b) the corresponding air-filled CMS

dotted lines represent the magnetic walls. The capacitance in the open boundary and the capacitance confined in the dielectric layer were calculated in this order, taking the finite thickness of the dielectric layer into account. For the capacitance confined in the dielectric layer, the boundary between the air and the dielectric layer is modeled as a magnetic wall and the dielectric layer is replaced by an air-filled capacitance, as shown in Fig. 6 (b).

The capacitance of the upper half-plane, region I and $\mathrm{I}$, and the capacitance of the finite thickness, region II and III, were computed exactly through the sequence of conformal mappings outlined in Fig. 7. The coordinates of regions I, II, III, and IV are transformed into an intermediate t-plane and then converted to a final w-plane. In the final plane, the strips are converted to a plane-parallel capacitor so that the capacitance can be easily evaluated.

The conformal mapping gives rise to analytical expressions for the capacitance in terms of the ratio of the complete elliptic integral of the first kind and its complement. The capacitance (in air) per unit length of the strip is:

$$
C_{i}^{a}=\frac{\varepsilon_{0}}{2} \frac{K\left(k_{i}^{\prime}\right)}{K\left(k_{i}\right)}(i=I, I I, I I I, I V)
$$

where 


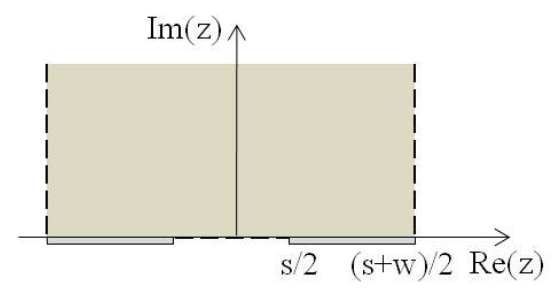

(a) Original structure of the open boundary

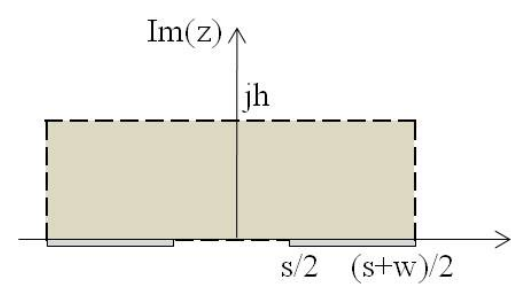

(b) Original structure of the enclosed boundary

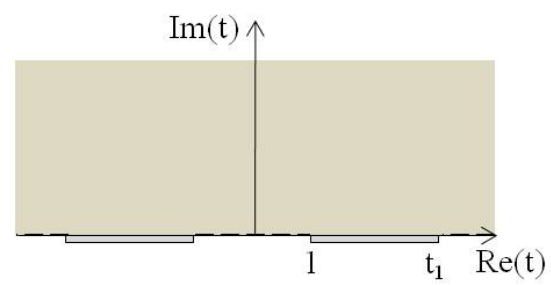

(c) The intermediate t-plane

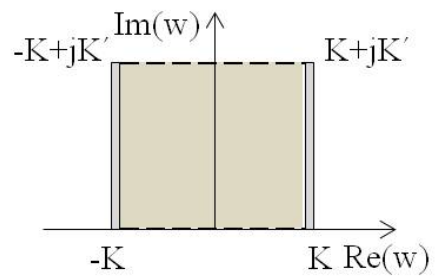

(d) The final mapping onto a plane-parallel capacitor

Fig. 7. Conformal mapping for the CMS structure.

$$
\begin{aligned}
& k_{I, I V}=\cos \left(\frac{w \pi}{2(w+s)}\right) \\
& k_{I I, I I I}=\operatorname{sn}\left(K\left(\varsigma_{i}^{\prime}\right) \frac{s}{2 w+s}, \varsigma_{i}^{\prime}\right) \\
& \varsigma_{I I}^{\prime}=\left(\frac{e^{\frac{(2 w+s) \pi}{2 h_{1}}}-2}{e^{\frac{(2 w+s) \pi}{2 h_{1}}}+2}\right)^{2} \\
& \varsigma_{I I I}^{\prime}=\left(\frac{e^{\frac{(2 w+s) \pi}{2 h_{2}}}-2}{e^{\frac{(2 w+s) \pi}{2 h_{2}}}+2}\right)^{2}
\end{aligned}
$$

$K(k)$ and $K\left(k^{\prime}\right)$ are the complete elliptic integral of the first kind and its complement, respectively, and $k$ ' is the complementary modulus. $s n$ is the Jacobi elliptic func- tion.

Accurate expressions for the ratio $K\left(k^{\prime}\right) / K(k)$ are available in [9]. These are given below:

$$
\begin{aligned}
& \frac{K\left(k^{\prime}\right)}{K(k)}=\frac{1}{\pi} \ln \left(2\left(1+\sqrt{k^{\prime}}\right) /\left(1-\sqrt{k^{\prime}}\right)\right) \\
& \frac{\text { for } 0 \leq k \leq \frac{1}{\sqrt{2}}}{K\left(k^{\prime}\right)}=\frac{\pi}{\ln (2(1+\sqrt{k}) /(1-\sqrt{k}))} \\
& \text { for } \frac{1}{\sqrt{2}} \leq k \leq 1
\end{aligned}
$$

The value of the filling factors can be written in terms of the variables above of the basic capacitances. The effective dielectric constant $\varepsilon_{\text {eff }}$ and the overall capacitance for the CMS can be obtained:

$$
\begin{aligned}
\varepsilon_{e f f}= & {\left[\left(C_{I}^{a}-C_{I I}^{a}\right)+\varepsilon_{r 1} C_{I I}^{a}+\varepsilon_{r 2} C_{I I I}^{a}\right.} \\
& \left.+\varepsilon_{r 4}\left(C_{I V}^{a}-C_{I I I}^{a}\right)\right] /\left(2 C_{I}^{a}\right) \\
C_{C M S} & =4 \varepsilon_{e f f} C_{I}^{a}
\end{aligned}
$$

The scaling of the metal conductivity and the surface impedance concept, which has proven to be an efficient method for accurately evaluating the frequency dependent series impedance of transmission lines [10], is used to evaluate the frequency dependent inductance and the resistance of the CMS. Based on the mapping above, the scaling factor for the CMS and its series impedance equation are given as follows:

$$
\begin{aligned}
& N(\xi)=\left|\sqrt{\left(\xi^{2}-1^{2}\right)\left(\xi^{2}-1 / k_{I}^{2}\right)}\right| \\
& M(\xi)=\left|\frac{-j \pi}{w+s} \sqrt{\left(\xi^{2}-1 / k_{I}^{2}\right)}\right| \\
& Z(\omega)=\left[2 \int_{1}^{1 / k_{I}} \frac{d \xi}{j \omega \mu_{0}\left|v_{t}-v_{b}\right| N(\xi)+2 Z s(\omega, \xi) M(\xi)}\right]^{-1}
\end{aligned}
$$

where

$$
\begin{aligned}
& \left|v_{t}-v_{b}\right|=2 k_{I} K\left(k_{I}\right) \\
& Z s(\omega, t, \sigma)=\frac{\frac{1}{\sigma \delta}(1+j)}{\tanh \left(\frac{t}{2 \delta}(1+j)\right)}
\end{aligned}
$$

$\delta$ is the skin depth and $\sigma$ and $t$ are the metal conductivity and the thickness of the metal, respectively.

The real term of the series impedance determines the resistive part, and the imaginary term determines the inductive part. 


$$
\begin{aligned}
& R_{C M S}(\omega)=\operatorname{Re}(Z(\omega)) \\
& L_{C M S}(\omega)=\operatorname{Im}(Z(\omega)) / \omega
\end{aligned}
$$

The RLC parameters of an air-filled CPW and CMS structure are compared in Fig. 8. The CPW and CMS structure are configured to have the same geometry, namely $w=25 \mu \mathrm{m}, s=25 \mu \mathrm{m}, t=0.525 \mu \mathrm{m}$, and $\sigma=$ $2.3 \times 10^{7}$. The graph confirms that the CMS structure requires dedicated equations to extract parameters because the parameters of the CMS are different than those of the CPW. The existence of the side magnetic wall causes the dissimilarities between the CMS and the CPW. As a result of the side magnetic walls, the CMS structure has a lower capacitance and a higher inductance and resistance compared to the CPW structure.

\section{Conductor-Backed Coplanar Multistrip}

The conductor-backed CMS resembles a mixed coplanar-microstrip structure. Microstrip behavior is known to become dominant when the dielectric between the signal and ground is thin and the slots are wide, such that $W \gg$ $h$ [11]. In a real meshed power/ground plane, the metal width and space are much wider than the dielectric thickness between the metals. Thus, in this paper, the ground strip in the coplane is neglected, and the conductor-backed CMS is treated as a microstrip. This assumption does not hold when the space between the horizontal coplane metal lines becomes so close that the fringing capacitance between metal lines is no longer negligible.

We evaluated the capacitance of the microstrip structure using an empirical expression based on the conformal mapping analysis in [12], which has become known as the most accurate formula. The maximum deviation from the analytical formula is given as 2 percent when $w / h \geq 1,0.1 \leq t / h \leq 4$ [13]. All cited ranges are adequate for typical chip applications.

$$
\begin{aligned}
& C_{\text {Micro }}^{a}=\varepsilon_{0}\left(\frac{w}{h}+0.77+1.06\left(\frac{w}{h}\right)^{0.25}+1.06\left(\frac{t}{h}\right)^{0.5}\right) \\
& C_{\text {Micro }}=\varepsilon_{r} C_{\text {Micro }}^{a}
\end{aligned}
$$

We evaluated the frequency dependent inductance and resistance using the approaches in [14] and [15]. At low frequencies, the current is uniformly distributed over the cross section of each conductor. As the frequency increases, the current distribution begins to change due to the induced electric field. In the low-frequency region, the closed-form expression for the inductance per unit length of the microstrip line is given by [14]:

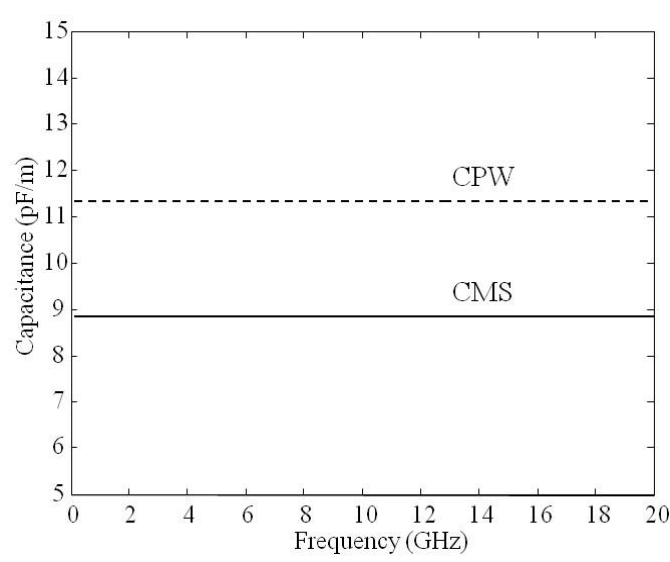

(a) Capacitance

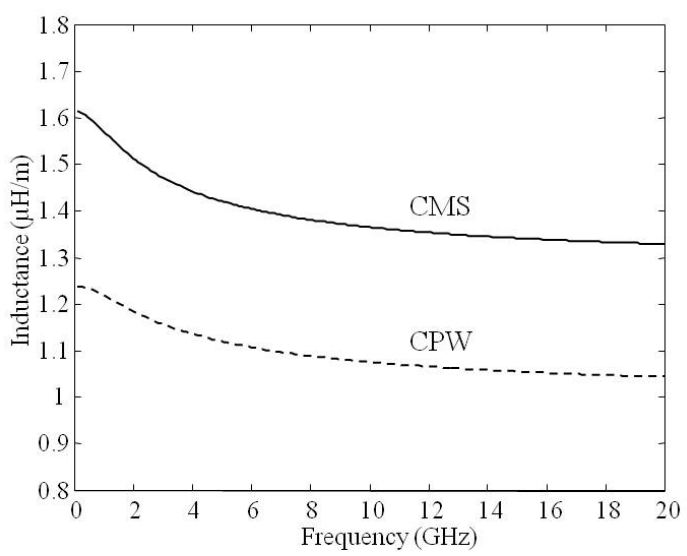

(b) Inductance

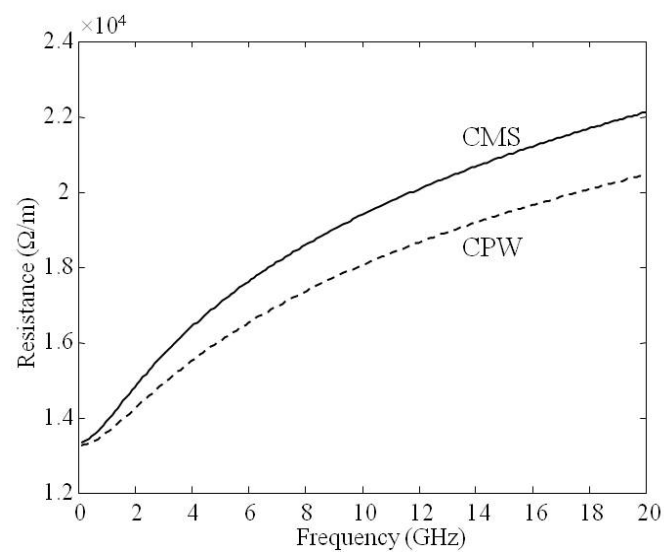

(c) Resistance

Fig. 8. Comparison of parameters for the CPW and the CMS.

$$
\begin{aligned}
& L_{D C}=-\frac{\mu_{0}}{2 \pi t^{2}}\left[\frac{1}{w^{2}} K_{s}(w, t)-\frac{2}{w \cdot w g}\right. \\
&\left.K_{m}(w, t, w g, t, h+t)+\frac{1}{w g^{2}} K_{s}(w g, t)\right]
\end{aligned}
$$

with 


$$
\begin{aligned}
& K_{s}(a, b) \\
& =\operatorname{Re}\{4 \cdot[K 4(a)+K 4(j b)]-2 \\
& \cdot[K 4(a+j b)+K 4(a-j b)]\}+\frac{1}{3} \pi \cdot a \cdot b^{3} \\
& K_{m}(a, b, c, d, h) \\
& =\operatorname{Re}\{-K 4(z 1+z 2-z 3-z 4-j h) \\
& \cdot \mid \begin{array}{c|c|c|c}
a / 2 & j b / 2 & c / 2 & j d / 2 \\
z 1=-a / 2 & z 2=-j b / 2 & z 3=-c / 2 & z 4=-j d / 2
\end{array} \\
& K 4(z)=\frac{z^{4}}{24}\left[\ln (z)-\frac{25}{12}\right]
\end{aligned}
$$

At high frequencies, the skin effects are so pronounced that the current becomes concentrated in a thin layer at the conductor surface. Thus, the inductance per unit length tends toward a constant, which can be expressed as:

$$
L_{\mathrm{inf}}=\varepsilon_{0} \mu_{0} \frac{1}{C_{\text {Micro }}^{a}}
$$

The resistance in the low frequencies, $R_{D C}$, consists of the resistance of the signal and that of the ground conductor:

$$
R_{D C}=R_{D C, w}+R_{D C, g}
$$

with

$$
\begin{aligned}
& R_{D C, w}=\frac{1}{\sigma \cdot w \cdot t} \\
& R_{D C, g}=\frac{1}{\sigma \cdot w g \cdot t}
\end{aligned}
$$

The resistance per unit length increases as the square root of the frequency due to the skin effect does. Hence:

$$
R_{s}(f)=\sqrt{\frac{\pi \mu_{0} f}{\sigma}}
$$

An approximate expression for the resistance per unit length in the high-frequency region is [14], [15]:

$$
\begin{aligned}
R_{\mathrm{inf}}(f) & =\frac{2}{h} \frac{Z_{\mathrm{inf}}^{a} R_{s}(f)}{\varsigma_{0}^{2}}\left\{\frac{w}{h}+\frac{6 h}{w}\left[\left(1-\frac{h}{w}\right)^{5}+0.08\right]\right\} \\
\cdot & {\left[1+\frac{h}{w}\left(1+\frac{1}{\pi} \ln \frac{2 h}{t}\right)\right] }
\end{aligned}
$$

where

$$
\begin{aligned}
& \varsigma_{0}=\sqrt{\frac{\mu_{0}}{\varepsilon_{0}}} \\
& Z_{\text {inf }}^{a}=\frac{\sqrt{\varepsilon_{0} \mu_{0}}}{C_{\text {micro }}^{a}}
\end{aligned}
$$

The resistance and inductance per unit length at an arbitrary frequency $f$ are approximated by the following equations. The metal thickness is usually less than 1 $\mu \mathrm{m}$, such that $f_{s}$ is considerably higher than the frequency of interest. The terms related to $f_{s}$ in the equations in [14] and [15] are removed for simplicity and better accuracy.

$$
\begin{aligned}
& L_{\text {Micro }}(f)=L_{D C}+\left(L_{D C}-L_{\mathrm{inf}}\right) / \sqrt{1+\left(\frac{f}{f_{0}}\right)^{2}} \\
& R_{\text {Micro }}(f)=R_{\mathrm{inf}}\left(f_{s}\right)-\left(R_{\mathrm{inf}}\left(f_{s}\right)-R_{D C}\right) / \sqrt{1+\left(\frac{f}{f_{0}}\right)^{2}}
\end{aligned}
$$

In these equations, $f_{o}$ and $f_{s}$ denote the frequency boundaries between the $\mathrm{dc}$ and intermediate ranges and between the intermediate and skin-effect ranges, respectively.

$$
\begin{aligned}
& f_{0}=\frac{2 \cdot R_{D C, w} \cdot R_{D C, g}}{\mu_{0} \cdot\left(R_{D C, w}+R_{D C, g}\right)} \\
& f_{s}=\frac{1.6+\frac{10 t / w}{1+w / h}}{1.2 \pi \mu_{0} \sigma t^{2}}
\end{aligned}
$$

The RLC parameters are calculated using equations (28) and (29) for several dimensions, and the results are shown in Fig. 9. The wider metal shows higher capacitance, lower inductance and lower resistance.

\section{Verification}

A test vehicle that has a meshed power/ground plane on a low-loss silicon substrate was simulated and modeled to verify the proposed model. Simulation was conducted using Ansoft HFSS, a 3D-field solver. For the model, the RLC parameters were calculated using Matlab and imported into a SPICE program, such as Agilent ADS. Thus, the whole on-chip PDN is composed of only lumped circuit parameters. The outline of the test vehicle is described in Fig. 10(a). The vertical dimensions and dielectric properties are shown in Fig. 10 (b), which is the typical stacking of a standard CMOS process. The entire dimension of the PDN was $1.5 \mathrm{~mm}$ by $1.8 \mathrm{~mm}$. The metal width was $20 \mu \mathrm{m}$, and the space was $30 \mu \mathrm{m}$. In the HFSS simulation, a unit cell of $300 \times 300 \mu \mathrm{m}$ was simulated and merged using a segmentation method because the simulation of a full onchip PDN is not readily available. Port 1 was assigned between power and the ground net at the center, port 2 at the edge, and port 3 at the corner of the co-chip PDN. 


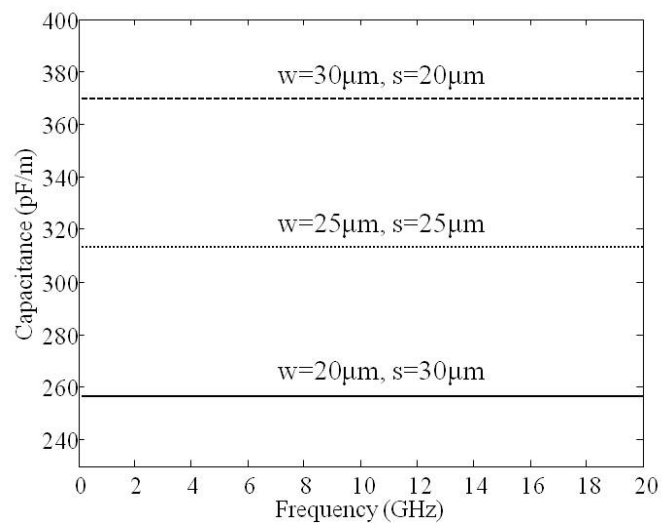

(a) Capacitance

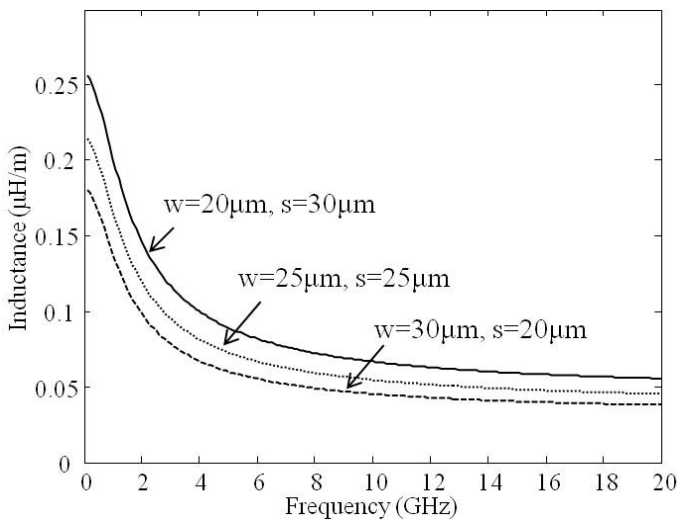

(b) Inductance

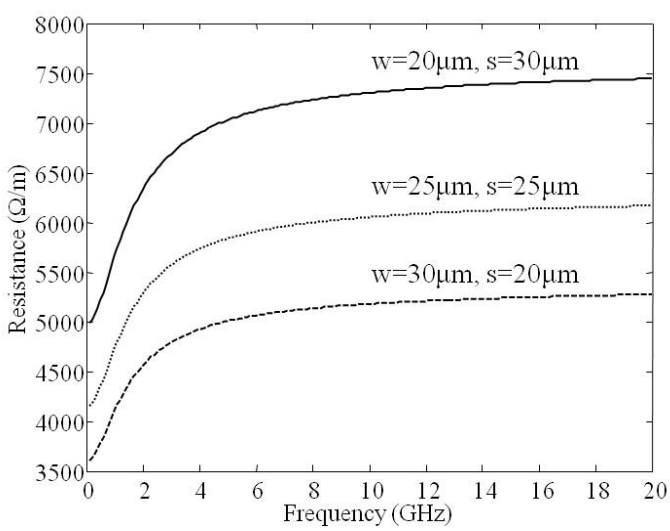

(c) Capacitance

Fig. 9. Comparison of the parameters for the CPW and the CMS.

The self-impedances at each port and the transfer impedances between ports are compared in Fig. 11. As with the PCB power/ground plane, series and parallel resonance phenomena appeared. Mode resonances cannot be clearly discriminated in an on-chip PDN because of the higher conductor loss compared to a PDN fabricated in PCB. Because of the different inductance seen at each port, the curves in Fig. 11(a) show different resonance frequencies. In Fig. 11(b), port 1 is located in

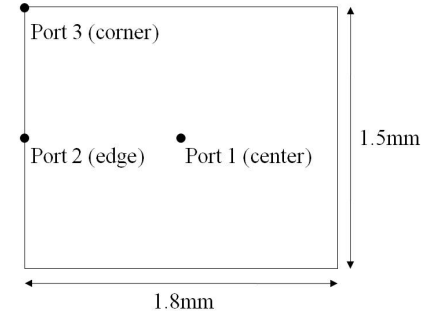

(a) PDN's size and port location

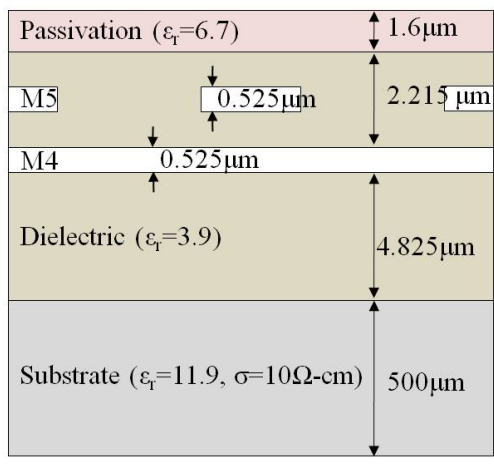

(b) Vertical dimensions and dielectric properties

Fig. 10. On-chip PDN configuration for the simulation and the model.

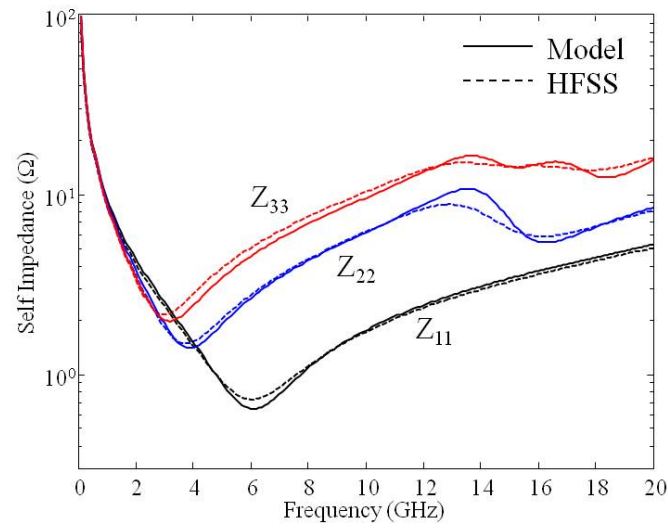

(a) Self-impedance

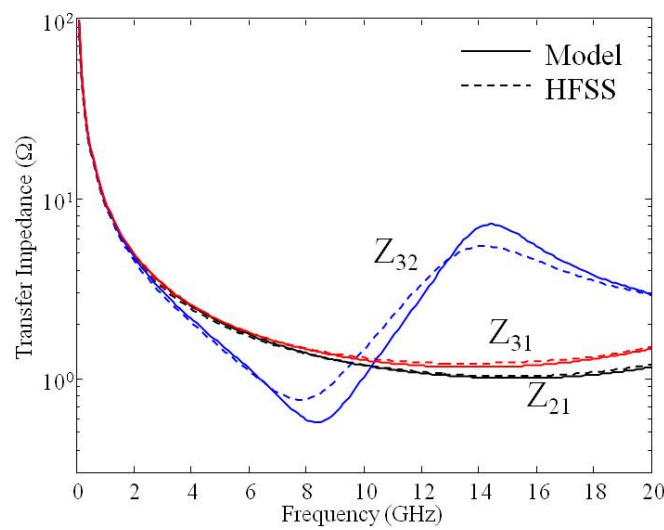

(b) Transfer impedance

Fig. 11. Correlation between the proposed model and the HFSS simulation. 
the center of the rectangular-shaped PDN; there is no $(0$, 1) or $(0,1)$ mode parallel resonance in the $Z_{31}$ and $Z_{21}$ curves. The graphs clearly show that the proposed model is well correlated with the results of HFSS for both self-impedances and transfer impedances.

\section{Conclusion}

For wideband impedance estimation, the on-chip power/ground meshed plane was modeled as a transmission line using frequency dependent $R, L$, and $C$ parameters. The meshed plane was divided into two cascaded transmission lines with uniform cross section for quasi-static analysis. For CMS structures, the frequency dependent $R, L$, and $C$ parameters were extracted using the proposed equations based on conformal mapping. The conductor-backed CMS structure was assumed to be that of a microstrip, and the previously developed methods were applied to extract the parameters. The proposed model for the on-chip power/ground meshed plane was validated by a comparison with the 3D-field solver, and it successfully estimated the characteristics of the on-chip power/ground meshed plane over a wide frequency range.

This work was supported by the IT R\&D program of MKE/KEIT. [KI001472, Wafer Level 3D IC Design and Integration]

\section{References}

[1] K. T. Tang, E. G. Friedman, "Simultaneous switching noise in on-chip CMOS power distribution networks," IEEE Trans. Very Large Scale Integration (VLSI) Systems, vol. 10, no. 4, pp. 487-493, Aug. 2002.

[2] M. Swaminathan, J. Kim, I. Novak, and J. P. Libous, "Power distribution networks for system-on-package: status and challenges," IEEE Trans. Adv. Packag., vol. 27, no. 2, pp. 286-300, May 2004.

[3] J. Choi, L. Wan, M. Swaminathan, B. Beker, and R. Master, "Modeling of realistic on-chip power grid using the FDTD method," IEEE International Symposium on Electromagnetic Compatibility, pp. 238243, 2002.

[4] J. Mao, W. Kim, S. Choi, M. Swaminathan, J. Li- bous, and D. O'connor, "Electromagnetic modelling of switching noise in on-chip power distribution networks," Proc. Int. Conf. Electromagnetic Interference and Compatibility (INCEMIC), pp. 47-52, 2003.

[5] J. Mao, "Modeling of simultaneous switching noise in on-chip and package power distribution networks using conformal mapping, finite difference time domain and cavity resonator methods," Ph. D. Dissertation. Georgia Institute of Technology, Aug. 2004.

[6] A. V. Mezhiba, E. G. Friedman, "Inductive properties of high-performance power distribution grids," IEEE Trans. Very Large Scale Integration (VLSI) Systems, vol. 10, no. 6, pp. 762-776, Dec. 2002.

[7] G. Ghione, C. Naldi, "Parameters of coplanar waveguides with lower ground plane," Electronics Letters, vol. 19, no. 18, pp. 734-735, Sep. 1983.

[8] C. P. Wen, "Coplanar-waveguide directional couplers," IEEE Trans. Microwave Theory Tech., vol. 18, no. 6, pp. 318-322, Jun. 1970.

[9] W. Hilberg, "From approximation to exact relations for characteristic impedances," IEEE Trans. Microwave Theory Tech., vol. 17, no. 5. pp. 259-265, May 1969.

[10] E. Tuncer, B. T. Lee, M. S. Islam, and D. P. Neikirk, "Quasi-static conductor loss calculations in transmission lines using a new conformal mapping technique," IEEE Trans. Microwave Theory Tech., vol. 42, no. 9, pp. 1807-1815, Sep. 1994.

[11] K. C. Gupta, R. Garg, I. Bahl, and P. Bhartia, Microstrip Lines and Slotlines, pp. 392-392, Artech House, 1996.

[12] N. v. d. Meijs, J. T. Fokkema, "VLSI circuit reconstruction from mask topology," Integration, vol. 2, no. 2, pp. 85-119, 1984.

[13] E. Barke, "Line-to-ground capacitance calculation for VLSI: a comparison," IEEE Trans. ComputerAided Design of Integrated Circuits and Systems, vol. 7, no. 2, pp. 295-298, Feb. 1988.

[14] A. R. Djordjevic, T. K. Sarkar, "Closed-form formulas for frequency-dependent resistance and inductance per unit length of microstrip and strip transmission lines," IEEE Trans. Microwave Theory Tech., vol. 42, no. 2, pp. 241-248, Feb. 1994.

[15] F. Schnieder, W. Heinrich, "Model of thin-film microstrip line for circuit design," IEEE Trans. Microwave Theory Tech., vol. 49, no. 1, pp. 104-110, Jan. 2001. 


\section{Chulsoon Hwang}

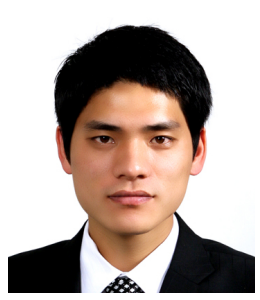

received B.S. and M.S. degrees in electrical engineering at the Korea Advanced Institute of Science and Technology (KAIST), Daejeon, Korea, in 2007 and 2009, respectively, where he is currently pursuing a $\mathrm{Ph} . \mathrm{D}$. degree in electrical engineering. His current research interests include the power integrity of chips/packages and suppression of power/ground noise coupling using electromagnetic bandgap (EBG) structures.

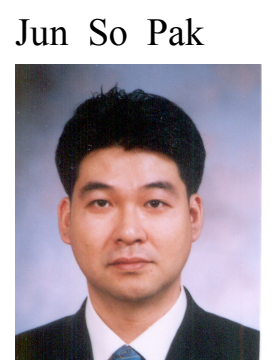

received a B.S. degree in electrical communication engineering from Hanyang University, Seoul, Korea, in 1998, and M.S. and $\mathrm{Ph} . \mathrm{D}$. degrees in electrical engineering from the Korea Advanced Institute of Science and Technology (KAIST), Daejeon, Korea, in 2000 and 2005, respectively. In 2005, he became a Research Fellow supported by JSPS in the High-Density Interconnection Group, AIST, Tsukuba, Japan, where he was involved in the development of interconnect techniques and interposers for 3-D multichip packaging. Since 2007, he has been a Research Professor of the Department of Electrical Engineering, KAIST. His current research interests are the development of a 3-D stacked chip package using through-silicon vias (TSVs).

Joungho Kim

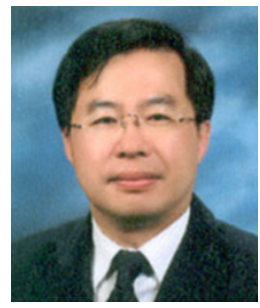

received B.S. and M.S. degrees in electrical engineering from Seoul National University, Seoul, Korea, in 1984 and 1986, respectively, and Ph.D. degree in electrical engineering from the University of Michigan, Ann Arbor, in 1993. In 1994, he joined Memory Division of Samsung Electronics, where he was engaged in Gbit-scale DRAM design. In 1996, he moved to KAIST (Korea Advanced Institute of Science and Technology). He is currently EECS Department Chair at KAIST. Since joining KAIST, his research centers on EMC modeling, design, and measurement methodologies of 3D IC, System-in- Package (SiP), multi-layer PCB, and wireless power transfer technology. Especially, his major research topic is focused on chippackage co-design and simulation for signal integrity, power integrity, ground integrity, timing integrity, and radiated emission of 3D IC and SiP. He has successfully demonstrated low noise and high performance designs of numerous SiP's for wireless communication applications such as ZigBee, T-DMB, NFC, and UWB. Recently, he started a new research on wireless power transfer technology using magnetic field resonance. He has been one of the co-leaders in a national project, OLEV (Online Electrical Vehicle), for EMI and EMF reduction design. The OLEV was selected as one of the 50 Best Inventions in 2010 by Times Magazine. He has authored and co-authored over 330 technical papers published at refereed journals and conference proceedings in modeling, design, and measurement of 3D IC, SiP, PCB, and wireless power transfer. Also, he has given more than 170 invited talks and tutorials at the academia and the related industries. 